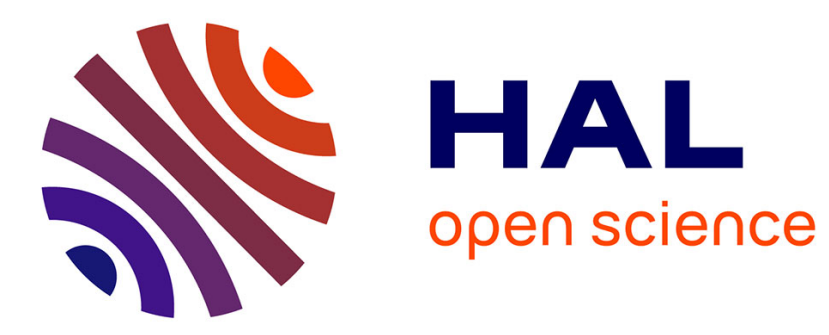

\title{
Le droit et ses histoires
}

Jean-Louis Halpérin

\section{To cite this version:}

Jean-Louis Halpérin. Le droit et ses histoires. Droit et Société, 2010, 75, pp.295-313. halshs-03281882

\section{HAL Id: halshs-03281882 \\ https://shs.hal.science/halshs-03281882}

Submitted on 8 Jul 2021

HAL is a multi-disciplinary open access archive for the deposit and dissemination of scientific research documents, whether they are published or not. The documents may come from teaching and research institutions in France or abroad, or from public or private research centers.
L'archive ouverte pluridisciplinaire HAL, est destinée au dépôt et à la diffusion de documents scientifiques de niveau recherche, publiés ou non, émanant des établissements d'enseignement et de recherche français ou étrangers, des laboratoires publics ou privés. 


\section{HEINONLINE}

DATE DOWNLOADED: Thu Jul 8 09:07:03 2021

SOURCE: Content Downloaded from HeinOnline

Citations:

Bluebook 21st ed.

Jean-Louis Halperin, Law and Its Histories, 75 DROIT eT Societe 295 (2010).

ALWD 6th ed.

Halperin, J. ., Law and Its Histories, 75 Droit et Societe 295 (2010).

APA 7th ed.

Halperin, J. (2010). Law and Its Histories. Droit et Societe, 75, 295-314.

Chicago 17th ed.

Jean-Louis Halperin, "Law and Its Histories," Droit et Societe 75 (2010): 295-314

McGill Guide 9th ed.

Jean-Louis Halperin, "Law and Its Histories" (2010) 75 Droit et Societe 295.

AGLC 4th ed.

Jean-Louis Halperin, 'Law and Its Histories' (2010) 75 Droit et Societe 295.

MLA 8th ed.

Halperin, Jean-Louis. "Law and Its Histories." Droit et Societe, 75, 2010, p.

295-314. HeinOnline.

OSCOLA 4th ed.

Jean-Louis Halperin, 'Law and Its Histories' (2010) 75 Droit et Societe 295

-- Your use of this HeinOnline PDF indicates your acceptance of HeinOnline's Terms and Conditions of the license agreement available at

https://heinonline.org/HOL/License

-- The search text of this PDF is generated from uncorrected OCR text.

-- To obtain permission to use this article beyond the scope of your license, please use: Copyright Information 


\title{
Le droit et ses histoires
}

\author{
Jean-Louis Halpérin
}

ENS-EHESS, Campus Paris-Jourdan, 48 boulevard Jourdan, F-75014 Paris.

<jean-louis.halperin@ens.fr>

Résumé Les historiens ont l'habitude de dire que le droit est histoire. En partant d'une approche positiviste, considérant que le droit est une technologie inventée dans des lieux et des moments différents, il paraît plus approprié d'affirmer que le droit a des histoires, variées en fonction des lectures des phénomènes du passé. Dans cette perspective, une des tâches de la méthode historique appliquée au droit est d'identifier et de comprendre les processus par lesquels la technologie juridique a été inventée, les ordres juridiques sont imposés ou déployés et les normes juridiques ont disparu ou paru battre en retraite. Au-delà de l'ordre juridique, la pluralité des aspects du champ juridique est la source inépuisable d'un grand nombre de différentes histoires du droit.

Histoire du droit - Invention du droit - Méthodologie juridique - Pluralisme - Règle de changement.

\section{Summary Law and its Histories}

Legal historians are accustomed to saying that law is history. Taking a positivistic approach and considering law as an invented technology (in different places and periods), it seems more convenient to affirm that law has histories, varying according to the interpretation of past phenomena. From this perspective, one of the tasks of an historical methodology applied to law is identifying and understanding the processes through which the legal technology is created, legal orders are imposed or displayed, and legal norms have disappeared or seemed to have beaten their retreat. Beyond the legal order, the plurality of aspects of the legal field is an inexhaustible source of a number of various legal histories.

Invention of law - Legal history - Legal methodology - Pluralism - Rule of change. 
L'historicité du droit fait figure de lieu commun depuis Savigny ${ }^{1}$. L'inscription des phénomènes juridiques dans le temps est pourtant l'objet de constants rappels de la part des historiens du droit, toujours inquiets de voir leur discipline marginalisée au nom d'une positivité limitée au droit en vigueur ou aux "prophéties » des décisions des tribunaux formant le droit à venir. S'il n'est guère difficile de montrer que même les règles d'aujourd'hui sont entrées dans l'ordre juridique à des dates différentes et sont destinées à en sortir un jour ou l'autre, il est plus délicat de justifier la méthode historique comme approche possible, voire souhaitable, de la connaissance du droit. Les ambitions de Savigny et de ses successeurs pour une science exclusivement historique du droit semblent depuis longtemps dépassées et victimes de la crise de l'historicisme.

Mus par une crainte, peut-être excessive, de voir leur discipline sombrer, même en tant que science auxiliaire, les historiens du droit nous paraissent sous-estimer les dangers que recèle leur habituelle profession de foi selon laquelle le droit est histoire $^{2}$. Non seulement cette identification laisse entendre que tout est historique dans le droit - voire que tout est juridique dans l'histoire -, mais elle induit l'existence d'un discours historique qui serait le méta-discours juridique. En répétant que le droit est histoire, ne laisse-t-on pas entendre que le développement du droit obéit à une Histoire, en passant par des phases articulées selon des schémas explicables? Apparaît alors la menace d'un retour à l'évolutionnisme si justement décrié sous toutes ses formes (histoire de l'Esprit, matérialisme historique, darwinisme social). De plus, une telle ardeur à vouloir “expliquer» les lois par l'histoire prête ouvertement le flanc à la critique kelsénienne d'une prétendue causalité qui chercherait en vain à voir dans les normes juridiques les effets de facteurs historiques. Si le rôle critique des historiens est de rappeler aux juristes ces facteurs déterminants - d'ordre politique, économique, social et culturel - qui pèsent sur la construction du droit, le même sens critique doit conduire au plus grand scepticisme sur une analyse univoque de la succession des phénomènes juridiques. Pour ne pas sombrer dans l'excès inverse - à quoi bon des études historiques sur le droit ?-, il nous paraît mieux en phase avec notre pratique professionnelle de dire que le droit "a des histoires ", qu'il est susceptible d'une pluralité de lectures prenant en compte l'inscription des phénomènes juridiques dans le temps et la diversité des tentatives pour “ écrire l'histoire » de ces phénomènes passés.

Plaidant depuis quelques années pour le remplacement de la traditionnelle " histoire des institutions "-élaborée le plus souvent dans une perspective nationale - par des histoires, à la fois séparables et liées entre elles, de la production normative, de la science du droit, des cultures juridiques ou des usages sociaux du droit ${ }^{3}$, nous voudrions mettre l'accent, à la faveur de ce dossier, sur les problèmes

1. Alfred Dufour, Droits de l'homme, droit naturel et histoire, Paris : PUF, coll. « Léviathan », 1991, p. 183.

2. Pio CARONI, «Blicke über den Gartenzaun. Von der Beziehung der Rechtsgechichte zu ihren historischen Nachbarwissenschaften», in Louis PAHLOw (Hrsg.), Die zeitliche Dimension des Rechts, Paderborn : Schöningh, 2005, p. 31.

3. Jean-Louis HaLPÊRIN, " Histoire du droit », in Denis AlLAND et Stéphane RIALs, Dictionnaire de la culture juridique, Paris : PUF, 2003, p. 787. 
historiques relatifs à l'invention du droit, à ses révolutions et à ses reculs ou retraits. Nous nous inscrivons résolument dans une perspective positiviste qui, en rejetant toute forme de droit naturel de caractère invariable (ce qui nous semble signifier une négation de l'histoire) ou à contenu changeant (ce qui constitue une forme d'aveu sur l'idéologie liée à toute conception d'un droit de la nature), conçoit le droit comme un artefact humain, une technologie 4 . Sur cette base positiviste, nous pensons qu'une des utilisations de la méthode historique consiste à identifier la présence ou l'absence des phénomènes juridiques dans un contexte social donné du passé, qu'il soit lointain ou proche. Â l'inverse de l'adage « ubi societas, ibi jus », que nous considérons antinomique avec le positivisme, nous estimons que les historiens du droit peuvent contribuer à une meilleure connaissance du droit en montrant que celui-ci n'est pas une donnée nécessaire, mais le résultat de processus de création (I). L'idée d'invention du droit suppose aussi une réversibilité historique : l'existence de moyens de destruction des règles et même des ordres juridiques $(I)$. Le refus de considérer que le droit est « déjà là », toujours présent et facile à mettre en lumière, nous amènera à revenir sur la pluralité des terrains auxquels sont susceptibles de s'appliquer des investigations historiques elles-mêmes marquées par une grande diversité de points de vue (III).

\section{L'étonnement devant les naissances du droit}

L'intérêt des historiens du droit pour les naissances du droit, dans l'optique de la différenciation des sources du droit ou dans celle de l'étude des processus de création des normes, n'est pas nouveau. Jean Gaudemet a donné ce titre à un manuel novateur qui entendait dépasser la traditionnelle « histoire externe » des sources du droit, " liste trop souvent monotone de lois, d'œuvres juridiques, de savants commentateurs $" 5$. Nous partageons cette conviction que la méthode historique est utile, sinon indispensable, à la compréhension de la dynamique du droit, à l'étude des processus de création des normes juridiques en incluant tout l'éventail des interprétations données aux énoncés normatifs. Il nous apparaît cependant nécessaire de ne pas s'en tenir à une lecture historique limitée aux «instantanés » des changements successifs intervenus à l'intérieur des ordres juridiques ${ }^{6}$, mais de porter aussi son attention sur l'établissement des ordres juridiques eux-mêmes. En défendant l'idée que le droit a été historiquement inventé en divers lieux et à des moments historiquement séparés, nous sommes logiquement amenés à revendiquer une approche historique adaptée à la mise en place d'ordres juridiques nouveaux.

4. Hans KELSEN, Théorie générale du droit et de l'État (trad. fr. Béatrice Laroche et Valérie Faure), Paris : LGDJ, coll. "La pensée juridique », 1977, p. 66-70 sur cette conception du droit comme « technique sociale spécifique».

5. Jean GAUDEMET, Les naissances du droit. Le temps, le pouvoir et la science au service du droit, Paris: Montchrestien, $2^{e}$ éd., 1999, p. VII.

6. François OsT, « L'instantané ou l'institution? L'institué ou l'instituant? Le droit a-t-il pour vocation de durer? ", in François OST et Mark VAN HOECKE (eds.), Temps et droit. Le droit a-t-il pour vocation de durer?, Bruxelles : Bruylant, 1998, p. 10. 


\section{I.1. L'historicité des inventions du droit}

L'ouvrage récent d'Aldo Schiavone sur l'invention du droit par les Romains a renouvelé la problématique historique sur le rôle fondateur de la civilisation romaine dans la tradition juridique occidentale ${ }^{7}$. L'auteur ne s'est pas contenté de reprendre et justifier l'idée ancienne selon laquelle les Romains ont été les premiers parmi les peuples méditerranéens à poser les fondements d'une « science du droit », il a insisté sur la rupture que constitue la rencontre entre la loi des XII Tables et le jus d'origine pontificale par rapport au nomos grec et plus encore par rapport aux suppositions habituellement faites à propos d'un droit mésopotamien ou égyptien. Partant de l'idée que nous projetterions sur les textes mésopotamiens ou égyptiens des conceptions du droit préformées par le droit romain ${ }^{8}$, nous avons défendu la thèse d'une absence de droit dans certaines civilisations antiques ou "primitives " et du caractère historiquement situé de l'invention du droit dans des civilisations séparées dans l'espace et n'entretenant pas nécessairement de liens entre elles 9.

Une telle thèse, ouverte à la discussion, nous paraît conforme aux postulats positivistes dégagés par Kelsen et Hart: le droit a besoin d'être distingué d'autres systèmes normatifs, seules les normes qui ont été en vigueur forment le droit, c'est le droit qui définit ses propres limites et permet d'identifier les normes juridiques, les ordres juridiques résultent de l'union de règles primaires (dont le contenu peut être semblable à celui d'autres systèmes normatifs de caractère religieux, social ou moral) et de règles secondaires (de reconnaissance, de changement et d'adjudication). Dans une perspective historique, nous insistons particulièrement sur le critère du changement. Face à des conceptions essentialistes, qui supposent des universaux ou des invariants dans le contenu des règles juridiques, nous affirmons que tout peut être changé dans un ordre juridique 10 et que la possibilité d'un changement - le fait que le changement soit en quelque sorte prévu d'avance et organisé par des règles procédurales - est précisément un critère déterminant pour identifier la création d'ordres juridiques par rapport à d'autres ordres de contrainte.

Une telle conception ravive l'intérêt pour des questionnements, qui ne sont heureusement pas susceptibles de réponses univoques, sur l'apparition historique des ordres juridiques. Si l'on admet que toute société n'a pas un droit, que l'ordre social et même politique peut être soutenu par des normes comportementales ne prenant pas la forme juridique, il est légitime de s'interroger sur le caractère juridique des systèmes normatifs des civilisations du Proche-Orient et de la Grèce archaïque.

Les débats restent, en effet, ouverts sur le caractère juridique du Code de Hammurabi, des "lois " pharaoniques ou du Code de Gortyne. S'agissant du premier

7. Aldo SCHIAvONE, Ius. L'invenzione del diritto in Occidente, Turin : Einaudi, 2005 ; ID., Ius. L'invention du droit en Occident, Paris : Belin, 2009.

8. À l'inverse de cette proposition d'Aldo Schiavone, Niklas LuHMANN (Law as a Social System, trad. Klaus Ziegert, Oxford : Oxford University Press, 2004) considérait qu'il fallait présumer que le droit n'avait pas d'origine et que les peuples anciens suivaient toujours des normes juridiques.

9. Jean-Louis HalPÉRIN, Profils des mondialisations du droit, Paris : Dalloz, 2009, p. 3-13.

10. Nous nous opposons sur ce point à Robert ALEXY, « Droit, discours et temps », in François OsT et Mark VAN HoECKE (eds.), Temps et droit, op. cit., p. 17. 
texte, connu de manière isolée de la pratique judiciaire ou administrative, les positions s'affrontent sur l'applicabilité de dispositions qui pourraient avoir un simple caractère programmatique. L'absence de référence à des règles antérieures, qui auraient pu être confirmées ou abrogées, et la disposition finale interdisant tout changement à l'avenir posent problème dans l'optique de l'union de règles primaires et secondaires 11 . Pour l'Égypte pharaonique, certains auteurs parlent d'édits royaux sans apporter de preuve que ces ordres du pharaon ont été reconnus, appliqués par des tribunaux et ont pu donner lieu à des changements. Les premiers textes connus qui répondent à ces critères remontent seulement à la période démotique (650-332 avant J.-C.) 12. Quant aux célèbres lois de Gortyne, connues par une monumentale inscription dont la plus grande partie date du $v^{e}$ siècle avant notre ère, elles surgissent mystérieusement dans une cité crétoise dont nous ne savons rien, dans un contexte de rareté de l'écriture ${ }^{13}$. Une interprétation possible est là aussi celle d'un monument, programme et discours destiné à frapper les esprits en prétendant à la permanence, et non d'un recueil de lois ayant été concrètement appliqué et amendé 14 . S'interrogeant plus largement sur les lois de la Grèce archaïque, Giorgio Camassa comprend la présence de clauses interdisant tout changement dans le texte écrit comme le témoignage d'une douloureuse invention du droit : pour insérer de telles clauses, les législateurs auraient eu conscience de la possibilité de changement qu'entraîne la technologie juridique associée à la mise par écrit (les règles orales de comportement peuvent changer, mais leur changement n'est pas daté ni facilement identifiable, comme le montre l'exemple des lois de Lycurgue restées orales pour assurer l'immutabilité de la société spartiate) et auraient cherché à préserver la stabilité par des anathèmes destinés à rester vains 15 .

Une telle lecture rejetant hors du droit des textes traditionnellement considérés comme ses premiers jalons au Proche-Orient et en Europe nous parait renforcer la thèse d'une invention du droit en Occident par les Romains. Au même siècle que le Code de Gortyne, la loi des XII Tables semble contenir une clause explicite sur une procédure de changement par l'assemblée du peuple et la tradition romaine rapporte que la prohibition des mariages entre patriciens et plébéiens, ajoutée tardivement par les décemvirs à la fin des XII tables, aurait été abrogée cinq années plus tard par la lex Canuleia. Ici l'union des règles primaires et secondaires, particulièrement celle de changement est visible et justifie le passage historique qu'évoquait Hart de règles pré-légales à des règles qualifiées de juridiques.

11. Martha T. RotH, «The Law Collection of King Hammurabi », in Edmond LÉvy (dir.), La Codification des lois dans l'Antiquité, Paris : De Boccard, 2000, p. 9-31.

12. Raymond WeStBrooK (ed.), A History of Ancient Near Eastern Law, La Haye, Boston : Brill, 2003, vol. I, p. $93,181,255,821$.

13. John DaviEs, «The Gortyn Laws », in Michael GaGarin et David Cohen (eds.), The Cambridge Companion to Ancient Greek Law, Cambridge : Cambridge University Press, 2005, p. 305-327.

14. James WhitLey, «Cretan Laws and Cretan Literacy », American Journal of Archeology, 101, 1997, p. 635661.

15. Giorgio CAMASSA, "Verschriftung und Veränderung der Gesetze» in Hans-Joachim GEHRKE (Hrsg-) Rechtskodifizierung und soziale Normen in interkulturellen Vergleich, Tübingen : G. Narr, 1994, p. 97-111. 
Deux objections viennent immédiatement à l'esprit face à cette conception (il s'agit nécessairement d'une interprétation ou construction historique) de l'invention du droit, supposant des sociétés " sans droit ». La première met en question la seule référence à des sources écrites, laissant entendre que le droit est l'apanage des civilisations de l'écriture. Il est vrai que la présence de la règle de changement ne paraît pas susceptible d'être prouvée par des restes archéologiques ne se présentant pas sous la forme d'inscriptions et que l'établissement d'une tradition orale de modifications apportées dans les règles de comportement a besoin d'un minimum de sources littéraires (même postérieures) qui peuvent rendre compte de cette oralité. L'historien nous paraît plus apte à chercher des témoignages d'un phénomène qui s'est produit dans le passé - par exemple, un changement dans une coutume orale attesté par une source écrite - qu'à postuler la présence nécessaire d'un phénomène en l'absence de preuve contraire. Faudrait-il conclure que toute société a un droit, faute de pouvoir démontrer un fait négatif, c'est-à-dire que l'absence de témoignage sur un droit signifie toujours l'ignorance de la technologie juridique?

La seconde objection qui a été déjà faite à cette perspective positiviste et historique de l'invention du droit est qu'elle constituerait un mythe ou une fable d'origine exclusivement occidentale projetant sur des civilisations anciennes ou « exotiques » des préjugés européo-centrés, voire racistes 16 . Nous y répondons au moyen de travaux historiques qui ont cherché à identifier, dans certains cas à travers des découvertes archéologiques récentes, des inventions du droit comparables en dehors de l'Occident et sans lien direct ou indirect avec la civilisation romaine. L'exemple chinois nous paraît le plus probant: face à une tradition littéraire qui attribuait, sans aucune référence précise, des lois aux premières dynasties à l'existence problématique plus de 2000 ans avant notre ère, les fouilles de tombes remontant à une période située entre le $\mathrm{IV}^{\mathrm{e}}$ et le $\mathrm{III}^{\mathrm{e}}$ siècle avant $\mathrm{J}$.-C. ont mis au jour des recueils de textes législatifs destinés à être appliqués par des fonctionnaires qui se posaient explicitement des questions d'abrogation des textes plus anciens par des lois postérieures ${ }^{17}$. Ces témoignages d'une grande précision sur l'invention de la technologie juridique corroborent une partie de la tradition littéraire remontant à Confucius qui associe à des « ducs» des codifications du droit dans leurs principautés, notamment les Qin, dont la montée en puissance aboutit à la formation du premier pouvoir impérial par Zeng de 221 à 210 avant J.-C. Or, ce sont justement les lois des Qin qui nous sont désormais connues par les planches de bambou des tombes du Hubei. Parmi les conseillers des princes Qin au IV siècle avant J.-C. figurent, de plus, les membres de l'École des Lois, notamment le célèbre Lang Shang 18. Comme à Rome, dans un contexte d'expansion territoriale - donnant lieu à une domination de type « impérial »- le pouvoir, probablement appuyé par des juristes,

16. Peter FitzPatrick, The Mythology of Modern Law, Abingdon : Routledge, 1992, notamment p. 192-197 sur l'histoire spéculative et la cosmologie occidentale attribuées aux conceptions de Hart du passage de règles pré-légales (règles primaires de comportement) aux normes juridiques intégrant les règles secondaires.

17. Yonping LIU, Origins of Chinese Law. Penal and Administrative Law in its Early Development, HongKong, Oxford, New York : Oxford University Press, 1998, p. 202.

18. The Book of Lord Shang (translat. J. J. L. Duvyvendok), Londres: A. Probsthain, 1928. 
joue un rôle moteur dans le recours à cette technologie du changement qu'est le droit.

Les exemples des droits juif et musulman nous montrent, eux aussi, que l'invention de la technologie du droit n'est pas l'apanage de l'Occident romanisé et que la règle de changement, comme les autres règles secondaires, n'est pas incompatible avec l'idée d'un législateur " divin ». Si le respect du texte de la Torah résulte du commandement de Dieu (Deutéronome 4, 2 : «Vous n’ajouterez rien à ce que je vous ordonne et vous n'en retrancherez rien, mais vous garderez les commandements de Yahvé votre Dieu tels que je vous les prescris »), le Talmud (traité Sanhedrin, 4,2 ) reconnaît qu'il n'y a pas de vérités préexistantes et définitives dans la loi juive, son interprétation dépendant de la majorité des interprètes à chaque génération 19. De même, l'intangibilité du Coran en tant que parole de Dieu ne concerne pas la totalité du droit musulman : la Sunna, formée des hadîth (paroles et actes attribués au Prophète), fait l'objet d'un examen historique qui a suscité de notables divergences entre les différentes écoles de juristes. L'activité intellectuelle (figh) de ces derniers a joué un rôle déterminant, particulièrement à la fin du vII siècle et au VIII ${ }^{e}$ siècle, pour fixer les hadith ${ }^{20}$. Ces juristes ont notamment développé des réflexions théoriques sur la notion d'abrogation ( naskh) pour résoudre les problèmes d'apparentes contradictions entre des versets du Coran, ou entre ces versets et les hadith, comme pour expliquer le maintien ou la suppression de coutumes préislamiques 21 . Si les droits religieux se fondent sur des dogmes éternels pour les croyants, ils laissent une part non négligeable à l'interprétation, voire au changement des règles multiples dont ils sont formés. L'invention du droit a ainsi pu se produire dans différentes civilisations, souvent bien après la découverte de l'écriture, et la démarche historique peut, selon nous, poser des questions pertinentes sur ces processus qui apparaissent liés à des phénomènes d'expansion des ordres juridiques en voie de constitution.

\section{I.2. L'historicité du déploiement des ordres juridiques}

Nous avons bien conscience que d'autres objections peuvent être présentées à une telle lecture qui dépend du sens donné au mot droit (en s'accrochant à la conception traditionnelle d'un « ensemble de règles»), paraît nécessiter l'existence d'un " législateur », voire d'un État, et semble négliger la création "spontanée " de normes juridiques venues « du bas », c'est-à-dire de forces sociales différenciées par rapport aux pouvoirs. Plus précisément, nous n'avons pas répondu à toutes les critiques adressées par Peter Fitzpatrick au schéma de Hart, accusé de reproduire le préjugé évolutionniste des Occidentaux d'un passage (qui aurait été provoqué,

19. Robert Goldenberg, "Preface", in Ben Zion Boksen et Baruch M. Bokser, The Talmud. Selected Writings, New York: Paulist Press, 1989, p. 11 et p. 27 avec l'exemple d'un changement d'interprétation sur la distance dont on peut s'éloigner de son domicile pendant le shabbat.

20. Hervé BLEUCHOT, Droit musulman. Essai d'approche anthropologique, Aix-en-Provence: Presses universitaires d'Aix-Marseille, 2000, t. I, p. 87, écrit ainsi que « le fiqh naît avant le hadith ».

21. Bernard G. WeIss, The Spirit of Islamic Law, Athènes, Londres: The University of Georgia Press, 2006, p. 90 . 
dans une partie du monde, par la colonisation) d'un état "pré-légal» au "monde du droit »22.

La démarche historique, qui suppose une réflexion sur les origines ou la généalogie des phénomènes, ne peut se dispenser d'une prise de position sur la différenciation entre les règles juridiques et les autres règles sociales de comportement. L'historien n'étant nullement obligé de souscrire à notre conception du positivisme juridique, il convient aussi d'envisager le recours à l'histoire dans d'autres cadres théoriques. S'il considère que le droit n'a pas d'existence en dehors du mythe (ce qui paraît être la position de Peter Fitzpatrick) ou des discours des juristes (dans une perspective souvent qualifiée de néo-positiviste), l'historien n'a apparemment pas besoin de s'interroger sur l'invention d'une technologie juridique par une autorité officielle, mais il lui reste à comprendre quand et comment ce mythe a été fabriqué, diffusé et imposé aux esprits. De même, si l'on refuse toute démarcation nette entre coutumes sociales et systèmes juridiques, il y a toujours lieu d'enquêter sur des situations historiques qui ont connu différents modes de régulation et de résolution des conflits.

Il ne s'agit pas, dans notre esprit, de rechercher et encore moins d'expliquer un «moment fondateur» du droit, mais de comprendre les processus ayant donné naissance à ce que nous appelons (depuis le XIX siècle) des "ordres juridiques" pensés - aujourd'hui encore, principalement dans des espaces étatiques et nationaux - comme des systèmes normatifs relativement clos sur eux-mêmes. Ces processus ont des histoires et les historiens qui adhèrent aux conceptions positivistes du droit donnent seulement plus de poids que d'autres au recours par des autorités politiques à une technologie juridique caractérisée par l'imposition de règles dites primaires et secondaires. Pour eux, le droit est le produit d'un pouvoir qui est en mesure non seulement d'établir des juges - c'est le critère de la "justiciabilité " utilisé par Kantorowicz ${ }^{23}$ et Kelsen -, mais aussi d'imaginer et de mettre en œuvre des procédures de changement des règles. Ce pouvoir peut être qualifié d'« État», comme l'a fait Kelsen, à condition de distinguer des ordres juridiques décentralisés au sein d'organisations politiques aux pouvoirs encore réduits (celles de la Rome républicaine, de la Chine des Qin ou des premiers califes musulmans) et des ordres juridiques centralisés correspondant à la naissance de "l'État moderne» dont l'avènement est souvent situé au XVI et au XVII siècle 24 .

Il n'y a pas de contradiction entre une telle démarche et l'étude de la construction, souvent parallèle, d'un discours juridique et d'une pensée du droit en tant que système, ordre ou mythe : tandis que le pouvoir utilise la technologie juridique, les « juristes » inventent l'ordre juridique assimilé à un peuple, un territoire ou un gou-

22. Peter FitzPATRICK, The Mythology of Modern Law, op. cit., p. 195. En sens contraire, Brian Z. TAMANAHA, "The Folly of the "Social Scientific" Concept of Legal Pluralism », Journal of Law and Society, 20, 1993, p. 192-212, relève, parmi d'autres critiques du pluralisme juridique auxquelles nous souscrivons, que le schéma de Hart a été adopté par de nombreux anthropologues.

23. Hermann Kantorowicz, The Definition of Law, Cambridge : Cambridge University Press, 1958, p. 78-79. 24. Jean-Louis HALPÉRIN, "De quelques difficultés kelséniennes pour l'historien du droit", in Denys DE BÉCHILLON, Pierre BRUNET, Véronique CHAMPEIL-DESPLATs et al. (études coordonnées par), L'architecture du droit. Mélanges en l'honneur du professeur Michel Troper, Paris : Economica, 2006, p. 477-486. 
vernement. L'histoire du droit hindou nous parait fournir un exemple révélateur des problèmes qui se posent aussi bien aux adeptes d'une conception dite pluraliste du droit qu'aux tenants du positivisme "classique». Tous s'accordent aujourd'hui à reconnaître que le "droit hindou » est, pour une grande part, une création des colonisateurs, notamment des Britanniques qui ont voulu voir, à partir de l'œuvre de William Jones ${ }^{25}$, la principale source historique de ce droit dans les "lois de Manou ». Or ce texte, daté dans une période qui va du II siècle avant J.-C. au II ${ }^{e}$ siècle de notre ère, n'est attribuable à aucun législateur (même collectif), n'a été mis en vigueur dans aucune des structures politiques de l'Inde antérieures à la conquête musulmane et ne parait jamais avoir été cité par les juges du Moyen Âge dont les décisions ont pu être retrouvées. Il y a donc lieu de se demander quel était le substrat du droit hindou avant l'arrivée des conquérants étrangers - à commencer par les sultans musulmans qui ont reconnu la loi personnelle des Hindous - et s'il avait un caractère juridique. Les tenants d'une vision pluraliste, refusant la démarcation occidentale entre règles coutumières et normes juridiques, en tirent argument pour y voir une autre conception du droit venu de la société qui n'aurait pas besoin de la consécration d'un pouvoir ou d'un législateur quelconque ${ }^{26}$. Nous sommes plus sensible, dans une perspective positiviste, au fait que s'est développé, au cours du Moyen Âge indien, une littérature spécialisée émanant d'auteurs dont le rôle (de " juristes"?) est d'expliquer les règles de comportement social sanctionnées par les tribunaux et susceptibles de plusieurs interprétations, voire de changements. Il y aurait ainsi un cas où la technologie juridique aurait été inventée par des juristes (ce qui vaut aussi partiellement pour le droit romain ou le droit musulman), sans impulsion donnée par un législateur ou une structure politique déterminée et stable. Nous serions ici en présence d'un processus de longue durée et non d'un passage daté des règles primaires à l'union de ces règles de comportement social avec les règles secondaires de reconnaissance, de changement et d'adjudication. Les conquérants musulmans, puis les colonisateurs britanniques, auraient transformé et accéléré ce processus en accréditant l'idée, diffusée par cette littérature, d'un droit hindou.

La recherche historique sur les naissances du droit ne peut se satisfaire de la croyance dans une apparition spontanée des règles juridiques : le droit né de la coutume est tout autant un mythe que le droit conçu de toutes pièces par un législateur herculéen. Les processus de déploiement des ordres juridiques sont extrêmement divers selon les contextes historiques, même s'ils font souvent appel à des facteurs communs comme l'établissement d'un pouvoir qui entend imposer un changement des règles sociales ou le développement d'une classe de spécialistes de l'interprétation de textes qui présenteraient une spécificité qualifiée d'une manière ou d'une autre de "juridique». Ce que nous appelons la "science du droit" en

25. David IBBETSON, "Sir William Jones as Comparative Lawyer " in Alexander MurRAY (ed.), Sir William Jones 1746-1794: A Commemoration (avec une introduction de Richard Gombrich), Clark (NJ) : The Lawbook Exchange, 2004, p. 13-28.

26. Werner F. MENSKI, Hindu Law. Beyond Tradition and Modernity, New Delhi : Oxford University Press, 2003, p. 78-106 
Occident, qui est apparue sous diverses formes dans l'espace et la chronologie, est peut-être séparable du droit qui en fait l'objet (c'est ce que pensent les positivistes), mais son essor est étroitement lié aux processus de développement des ordres juridiques. Ainsi la renaissance du droit romain en Occident, à partir du XII siècle et de l'épicentre bolognais, constitue sans conteste une « révolution juridique » 27 portée par les juristes, mais supposant aussi des substrats politiques et idéologiques, comme l'intervention de pouvoirs (la Papauté, les royaumes dans lesquels apparaissent les racines de l'État moderne, les communes qui se dotent de statuts...). Chacune des révolutions, qui a prétendu faire table rase du passé (la révolution américaine, la Révolution française, la révolution bolchevique, la révolution chinoise, mais aussi l'indépendance des États jusque-là colonisés), peut être analysée comme la naissance d'un ordre juridique nouveau qui pose aussi des questions aux historiens sur la création massive de nouvelles normes intégrées par la science du droit dans un système national - par exemple, pour concevoir un droit américain, un droit soviétique ou un droit indien. S'interroger sur la naissance de la technologie juridique ou la construction de normes juridiques conduit à poser la problématique des « disparitions $»$ du droit.

\section{Les interrogations sur les disparitions du droit}

En insistant sur le critère du changement pour la détermination des ordres juridiques, la perspective historique met en valeur leur métamorphose, à la fois perpétuelle et susceptible de rythmes ou de formes très variables. Comme le soulignait Jellinek, l'évolution de tout ordre juridique peut être analysée - à titre métaphorique, faut-il le rappeler? - comme une lutte entre un ancien et un nouveau droit 28 . Les juristes devraient, à l'instar de Jhering comparant le droit à Saturne dévorant ses propres enfants ${ }^{29}$, se résigner à cette instabilité de droits en dépit des objectifs d'ordre et de permanence. Mais ils sont souvent tentés de plaider la continuité des institutions, sinon des règles, ou de disqualifier les changements dans le contenu des normes en y voyant un « déclin du Droit » 30 . Non seulement les historiens du droit sont à même de rejeter un tel jugement de valeur supposant une essence atemporelle du droit, à laquelle le prétendu mouvement de déclin pourrait porter atteinte, mais leurs recherches des différences entre les droits du présent et ceux du passé peuvent s'enrichir d'une attention particulière aux phénomènes de succession des ordres juridiques dans le temps et de "retraits » du droit dans certains domaines ou contextes. Parmi les histoires juridiques, celles de la disparition, dans

27. Harold J. BERMAN, Law and Revolution. The Formation of the Western Legal Tradition, Cambridge (Mass.) : Harvard University Press, 1983, p. 11 sur l'apparition du droit comme un processus, une entreprise donnant naissance à un système intégré.

28. Georg JELLINEK, Der Kampf des alten mit dem neuen Recht», Heidelberg, 1907, ID., Ausgewählte Schriften und Reden, Berlin, 1911, p. 392-427.

29. Rudolf VON JHERING, L'esprit du droit romain (trad. O. de Meulenaere), 1886-1888, t. II, liv. II, \$ 32, rééd. Bologne : Forni, 1969, p. 69.

30. Georges RIPERT, Le déclin du droit : études sur la législation contemporaine, Paris: LGDJ, 1949. 
certains cas massive, des normes ont autant d'intérêt que celles de la naissance de la technologie juridique.

\section{II.1. Variété des successions d'ordres juridiques}

Dans le déploiement des ordres juridiques, l'importance des révolutions a déjà été soulignée. L'on pense, d'abord, aux changements brusques et plus ou moins violents de régime politique qui s'accompagnent d'une nouvelle constitution, d'une vaste refonte de la législation, de la formation (ou éventuellement de la disparition) d'un État indépendant prenant la place d'une domination coloniale et souvent de bouleversements profonds des professions et mentalités juridiques par une volonté de rupture avec "l'Ancien Régime». Les historiens du droit sont amenés à constater, si du moins ils portent leurs regards vers ces révolutions, la complexité de ces substitutions rapides d'un ordre juridique à un autre. La volonté de "table rase " des Insurgents américains, qui souhaitaient au départ se débarrasser du common law anglais, comme celle des révolutionnaires français de 1789, qui rêvaient de légiférer de nouveau sur l'ensemble du droit, s'est heurtée aux difficultés de production d'un arsenal législatif abrogeant tous les textes antérieurs : de ce fait, l'ancien droit a été maintenu chaque fois qu'il n'était pas rapporté ${ }^{31}$. La révolution bolchevique a, en revanche, procédé d'un coup à la suppression de tout le droit tsariste ${ }^{32}$, de même que la révolution chinoise en 1949. Au Xx $x^{e}$ siècle, les processus de décolonisation se sont plutôt accompagnés du maintien, au moins à titre provisoire, de normes d'origine coloniale, même si certains pays ont fait triompher leur législation nouvelle sur la pluralité des statuts personnels. À chaque fois des enquêtes historiques sont encore à faire pour mieux comprendre ces moments forts de transformations du droit et la disparition, effective ou non, des normes préexistantes à la révolution.

Les liens opérés entre le déploiement des ordres juridiques et leurs représentations, notamment au sein de la doctrine ou plus globalement de la science du droit, suscitent également des questionnements historiques sur les révolutions scientifiques, assimilées depuis les travaux de Thomas Kuhn aux "changements de paradigme » 33 . La renaissance du droit romain, à partir de Bologne à la fin du XI ${ }^{e}$ siècle, constitue l'une de ces révolutions juridiques qui a rendu au droit de Justinien un rôle éminent de "moment de validité " ou d'étalon juridique ${ }^{34}$. De plus en plus de chercheurs se demandent si l'émergence du droit coutumier à partir du XIII siècle,

31. Dans le cas américain, comme dans le cas français, la mythologie de la table rase s'est accompagnée de propositions de suppression du recours aux professionnels du droit: Lawrence M. FRIEDMAN, A History of American Law, New York: Simon and Schuster, 1973, p. 113 ; Jean-Louis HALPÉRIN, « Haro sur les hommes de loi », Droits, 17, 1993, p. 55-65.

32. Samuel Kucherov, The Organs of Soviet Administration of Justice: Their History and Operation, La Haye : E. J. Brill, 1970. En Chine, la rupture juridique a nécessité quelques mois ou quelques années, par exemple pour reformer le droit du mariage en 1950 : Michael PALMER, « The Re-emergence of Family Law in Post-Mao China : Marriage, Divorce and Reproduction », The China Quarterly, 141, 1995, p. 110-134.

33. Thomas Samuel KuHN, La structure des révolutions scientifiques (titre original : The Structure of Scientific Revolutions), Paris : Flammarion, 1972.

34. Paolo Grossi, L'ordine giuridico medievale, Roma-Bari : Laterza, 1996, p. 160 
traditionnellement considérée comme la "cristallisation » d'un droit spontané, n'est pas un effet réflexe et innovant du développement du droit savant ${ }^{35}$. La question de savoir si l'humanisme juridique du XvI siècle a provoqué, en parallèle avec la construction des États modernes, une révolution dans l'emploi des méthodes propres au «jus commune» avec la mise en place progressive d'ordres juridiques nationaux est plus controversée ${ }^{36}$. De même les débats sur l'éventuel passage sous l'influence des doctrines venues du continent (l'École du droit naturel moderne, mais aussi Pothier et plus tard Savigny) et à travers les travaux de Blackstone, Bentham, Austin et Dicey - d'un « vieux » common law à un droit anglais repensé et substantiellement transformé par la législation des XVIII et XIX siècles ${ }^{37}$, comme sur un possible "changement de paradigme" dans l'interprétation de la codification napoléonienne au cours du XIX ${ }^{e}$ siècle en France ${ }^{38}$, posent le problème de l'appréciation d'un basculement normatif et conceptuel qui ferait disparaître d'anciennes normes au profit de nouvelles. Les révolutions culturelles sont à la fois mieux préparées et plus lentes que les révolutions politiques. L'étude historique de l'abrogation ou de la désuétude des normes juridiques - ce qui s'applique aussi aux changements dans l'interprétation authentique d'énoncés normatifs apparemment maintenus dans l'état - stimule l'attention des chercheurs pour découvrir de fréquents décalages entre les ordres juridiques empiriques et la perception qu'en ont les contemporains.

\section{II.2. Ambiguité des « retraits » du droit}

Il est d'autres formes de disparition des normes juridiques dans un secteur donné que les historiens du droit cherchent à mettre en valeur, toujours avec cette idée que les moments paroxystiques de changement, les crises (au sens de la statis grecque qui est aussi «état»), sont révélateurs pour comprendre les processus de création des normes et, même au-delà, leur vie "normale " dans des circonstances plus apaisées. En dehors des phénomènes proprement révolutionnaires, plusieurs configurations historiques montrent un « retrait » du droit, l'effacement de normes anciennes qui encadraient tel ou tel comportement, ouvrant la voie à davantage de liberté ou au contraire de violence. Les historiens du droit pénal sont habitués à de tels déplacements des incriminations, dont beaucoup ont cessé d'exister ou d'être sanctionnées après avoir joué un rôle fondamental pendant des siècles. La décriminalisation ou dépénalisation de l'hérésie, du sacrilège, du blasphème, de la magie, de l'adultère, de l'avortement, de l'homosexualité sont autant de processus

35. Robert JACOB, "Les coutumiers du XII ${ }^{e}$ siècle ont-ils connu la coutume? ", in Mireille MousNIER et Jacques PoumarÈDE, La coutume au village dans l'Europe médiévale et moderne, Toulouse: Presses universitaires du Mirail, 2001, p. 103-119.

36. Marie-France RENOUX-ZAGAMÉ, « La méthode du droit commun : réflexions sur la logique des droits non codifiés ", Revue d'histoire des facultés de droit et de la science juridique, 10/11, 1990, p. 133-152.

37. Michael LoBBAN, The Common Law and English Jurisprudence 1760-1850, Oxford : Clatendon Press, 1991 ; David IBBEISON, A Historical Introduction to the Law of Obligations, Oxford : Oxford University Press, 1999 ; Denis BaRANGER, Écrire la constitution non écrite, Paris : PUF, coll. « Léviathan », 2008.

38. Alfons BÜRGE, "Le Code civil et son évolution vers un droit imprégné d'individualisme libéral », Revue trimestrielle de droit civil, janv.-mars 2000, p. 1-24. 
juridiques qui ont conduit à faire tomber des pans entiers du droit pénal et, quand le relais n'est pas pris par d'autres branches du droit (par exemple, le droit de la liberté de pensée ou de la lutte contre les discriminations), à laisser à d'autres modes de régulation sociale des domaines jusque-là réglés par le droit. Une telle « retraite » d'un législateur qui se veut plus modeste, en apparence plus respectueux des croyances et de la vie privée, peut être historiquement relativisée, notamment si l'on suit les thèses de Michel Foucault sur les progrès du contrôle social aux dépens du droit.

Une autre forme de retrait, souvent plus discret, intervient dans des contextes sociaux différents quand les autorités laissent les parties régler elles-mêmes leurs conflits, en diminuant la pression sur l'application effective des normes juridiques. Les travaux en langue anglaise qui ont mis en valeur des situations où l'ordre parvient à s'imposer sans le recours au droit ${ }^{39}$ nous paraissent utiles pour rappeler juristes et historiens du droit à la modestie par rapport à un préjugé courant d'omnipotence du droit. Dans les cas étudiés, ceux des éleveurs de bétail dans un comté de Californie ou des communautés Quakers, l'absence de recours aux tribunaux ou l'application de règles étrangères au droit ne signifie pas, pour autant, le retranchement des personnes considérées en dehors des ordres juridiques nationaux auxquels elles appartiennent. À nouveau, l'apparent mouvement de retrait du droit, analysé dans un contexte particulier, ne correspond aucunement à un retour vers un état "pré-juridique». L'abstention, à vrai dire limitée, des juges ou des professionnels du droit, est tolérée, sinon organisée par l'ordre juridique lui-même. L'on sait aussi, grâce aux travaux de Michel Foucault, combien les «illégalismes " peuvent relever d'une prévision du législateur.

Autrement tragiques sont toutes les situations historiques où, dans le cadre d'États autoritaires mais aussi d'États s'affirmant démocratiques confrontés à des situations exceptionnelles 40 ou à des populations jugées marginales 41 , la violence étatique a provoqué une complète retraite du droit. L'histoire du droit ne doit pas négliger, bien au contraire, ces frontières sanglantes du droit. Le régime nazi ne peut ainsi être écarté de l'enquête juridique, au motif qu'il aurait détruit toutes les normes de droit avec la solution finale : il a plutôt fait coexister, de manière monstrueuse, un ordre juridique allemand maintenu dans plusieurs de ses bases antérieures (comme le BGB interprété à l'aune de la législation antisémite dans les procès entre Aryens et Juifs) avec des procédures d'élimination systématique qui échappaient au droit en étant secrètes, en reposant sur des ordres verbaux (pour l'euthanasie des malades mentaux ${ }^{42}$ ), et en ne laissant aucun moyen de recours aux victimes. Le droit et les professionnels qui en vivent ne paraissent pas suscepti-

39. Robert C. ElLICKSON, Order Without Law: How Neighbors Settle Disputes, Cambidge (Mass.) : Harvard University Press, 1991 ; Anthony BRADNEY et Fiona COWNIE, Living Without Law, Ashgate : Dartmouth, 2000.

40. François SAINT-BonNET, L'état d'exception, Paris : PUF, coll. « Droit fondamental », 2001.

41. À titre d'exemple pour la France, Alain DEWERPE, Charonne, 8 février 1962: anthropologie historique d'un massacre d'État, Paris : Gallimard, 2006.

42. Lothar GRUCHMANN, Justiz im Dritten Reich, Munich : Oldenbourg, 2001 montre les tensions entre le ministère de la Justice qui essaya de réformer le Code pénal et les ordres d'Hitler sur l'euthanasie des malades mentaux. 
bles de disparaître facilement, une fois la technologie juridique inventée. Ainsi, les histoires des ruptures juridiques nous ramènent, sans croyance dans une quelconque permanence de la Justice, aux régularités du droit qui forment le champ commun à l'histoire et à la sociologie.

\section{De la pluralité des terrains au kaléidoscope des approches}

Quand ils ont plaidé en faveur du recours à l'histoire, les théoriciens du droit ont généralement postulé l'existence d'une méthode historique, opposée à la dogmatique classique des juristes, dans la mesure où elle tenait compte des faits et de leur évolution (Saleilles ${ }^{43}$ ), s'intéressait aux effets empiriques des règles (Weber ${ }^{44}$ ) ou étudiait toutes les interprétations données à un énoncé normatif sans chercher à en privilégier une pour régler un conflit contemporain (Gadamer ${ }^{45}$ ). Nous sommes plutôt tenté de dire que les historiens du droit n'ont pas de méthode qui leur soit propre, qu'ils cherchent comme tous les historiens à comprendre les phénomènes du passé. La méthode historique n'a pas à s'adapter à l'étude des phénomènes juridiques passés, mais elle est susceptible de se décliner selon un grand nombre de modalités - et non en suivant une Histoire du droit univoque - en raison de la pluralité des objets liés au droit et de la variété des points de vue des observateurs. La discontinuité introduite par les idées de naissance et de retrait du droit se combine avec la multiplicité des lectures historiques du droit : parce que le droit a connu un éventail d'histoires ne pouvant toutes être parcourues en même temps (à supposer qu'elles puissent être atteintes dans leur intégralité à travers les témoignages conservés), il suscite une infinité d'écritures de ses histoires.

\section{1. Diversité des objets de l'histoire du droit}

Nous avons déjà relevé combien les schémas évolutionnistes avaient pesé sur le développement de l'histoire du droit en tant que discipline universitaire au XIX siècle 46 . Le postulat selon lequel toute société avait un droit, la croyance dans un progrès vers un droit de plus en plus rationnel et complexe, la conviction que les ordres juridiques étaient modelés par la conscience populaire de chaque nation (selon les thèses de l'École historique du droit en Allemagne) ou par l'action de l'État (selon l'opinion des historiens classiques du droit français), tout a concouru à accréditer l'idée d'une histoire nationale du droit relativement univoque. Les préjugés jusnaturalistes ont pesé de leur poids pour présenter, de manière relativement indifférenciée, les contributions respectives de la législation, de la coutume,

43. Raymond SALEILLES, "Quelques mots sur le rôle de la méthode historique dans l'enseignement du droit ", Revue internationale de l'enseignement, 1890, p. 482-503.

44. Max WEBER, Rudolf Stammler et le matérialisme historique: aux origines de la sociologie wébérienne (traduit de l'allemand par Michel Coutu et Dominique Leydet, avec la collaboration de Guy Rocher et Elke Winter), Paris : éd. du Cerf, Sainte-Foy (Québec) : Presses de l'Université Laval, 2001, p. 63 et 145.

45. Hans-Georg GADAMER, Vérité et méthode : les grandes lignes d'une herméneutique philosophique (traduit par Étienne Sacre et Paul Ricoeur), Paris : Seuil, 1976, p. 168 et suiv.

46. Jean-Louis HALPÉRIN, «L'histoire du droit constituée en discipline : consécration ou repli identitaire? ", Revue d'histoire des sciences humaines, 4, 2001, p. 9-32. 
de la jurisprudence et de la doctrine à cette œuvre de construction d'un droit national. Dans le cas de la France, l'État moderne et républicain a été pensé par les historiens du droit - qu'ils soient ardemment favorables à la République (comme Esmein) ou plutôt nostalgiques de l'Ancien Régime (comme Chénon, Declareuil ou Olivier-Martin) - comme le prolongement de l'État monarchique bâti en réaction au morcellement féodal. De manière encore plus nette, l'histoire du droit privé français du Moyen Âge au XVIII siècle a été systématiquement décrite comme une marche inéluctable vers l'unification et la codification, en négligeant les forces qui s'opposaient à cette évolution, sous l'Ancien Régime et même sous la Révolution. La notion d'institutions, supposant que les relations juridiques réunies en un faisceau sous un certain nombre d'idées avaient une sorte de vie organique à travers les siècles, a aussi ancré dans l'esprit d'un grand nombre d'historiens cette illusion d'une ligne cohérente permettant de réunir des points apparemment isolés dans l'histoire. En Allemagne, la traditionnelle histoire des concepts ou dogmes juridiques (Dogmengeschichte) a de même accrédité l'idée d'une histoire du droit abstraite et continuiste, aujourd'hui remise en cause 47 .

La montée en puissance d'une plus grande rigueur positiviste - appelant au minimum une distinction entre les règles de droit et la science du droit -, la méfiance à l'égard de tout évolutionnisme anthropomorphique, une meilleure prise en compte des contextes économiques et sociaux, l'emploi de méthodes quantitatives dans l'analyse des discours, l'influence de la sociologie des professions ou des phénomènes culturels sont, en sens inverse, autant de facteurs de nature à favoriser aujourd'hui une reconnaissance de la diversité des objets qui peuvent ou doivent retenir l'attention des historiens du droit. La dichotomie positiviste droit/science du droit entraîne déjà avec elle un souci de mieux distinguer les transformations des normes et celles des opinions des juristes, notamment de la doctrine, relativement à ces normes. L'attention portée aux critères de différenciation entre les règles juridiques et les autres ordres de régulation ou de contrainte sociale s'accompagne d'une plus grande sensibilité à l'égard de la complexité des "champs juridiques " dans lesquels s'inscrivent les ordres juridiques. Les analyses de Pierre Bourdieu sur la compétition qui s'exerce dans un champ juridique historiquement donné entre différentes communautés professionnelles de juristes invitent à mettre en relation l'histoire des professions juridiques, celle de l'édiction des normes et du pouvoir qui est reconnu à leurs interprètes 48 . Mieux conscient du fait que ses matériaux - des textes du passé relatifs au droit - n'ont pas tous constitué ou rapporté du droit positif, l'historien du droit cherche à comprendre comment fonctionnait, à une époque donnée, un champ juridique qui ne se limite pas aux règles en vigueur à un moment déterminé et n'est pas séparé d'autres pratiques sociales.

47. Johannes Michael SCHOLZ, Vorstudien zur Rechtshistorik, Francfort/Main : Vittorio Klostermann, coll " Jus commune », 1977 ; Jochen Hoock, " Dimension analytique et herméneutique d'une histoire historienne du droit », Annales ESC, 44 (6), 1989, p. 1479-1490.

48. Pierre Bourdieu, «La force du droit. Éléments pour une sociologie du champ juridique », Actes de la recherche en sciences sociales, 64, 1986, p. 3-19; Jacques CAILLOSSE, "Pierre Bourdieu, juris lector: antijuridisme et science du droit », Droit et Société, 56/57, 2004, p. 17-39. 
S'ouvrent alors d'infinies possibilités de différencier une histoire normative de la production des règles de droit (centrée sur les modes de création, de changement ou d'abrogation des normes par les autorités habilitées à décider du droit positif), une histoire de la science du droit (consacrée aux productions savantes, à l'enseignement du droit, aux influences intellectuelles entre juristes ou avec d'autres penseurs), une histoire des cultures juridiques (propre à chaque communauté professionnelle de juristes, voire à la population des profanes en droit), une histoire des pratiques (relative aux actes juridiques en apparence routiniers, comme les jugements ou les contrats, ainsi qu'aux régularités dans les usages du droit). Chacune de ces histoires peut s'attacher à un objet spécifique dans le temps, dans l'espace et dans les matières affectées par le droit. D'une affaire particulière peut naître une réflexion sur l'histoire juridique d'une notion ${ }^{49}$. Sans doute est-il vain de croire que la réunion de ces différentes " tranches " historiques suffirait à restituer l'intégralité de l'évolution d'un champ juridique d'une époque à une autre, mais il ne fait guère de doute que chaque strate peut aider à mieux comprendre les autres. Même avec le scepticisme le plus kelsénien par rapport à l'influence de la doctrine sur la création des normes juridiques, l'histoire de la science du droit n'est pas dépourvue d'intérêt pour décrire les processus de développement des ordres juridiques. La manière dont les romanistes du Moyen Âge ont réussi à convaincre les pouvoirs en place de la "validité " des textes de la compilation de Justinien et des interprétations dont ils faisaient l'objet est, sans aucun doute, décisive dans la compréhension de la réception du droit romain en tant que « raison écrite» douée de positivité dans une grande partie de l'Europe.

Comme les juristes attachent en général une grande importance à la tradition et aux précédents, la science, l'enseignement et dans une large mesure les processus d'interprétation du droit gardent la mémoire de faits ou de discours antérieurs. Loin de signifier l'immobilisme du champ juridique, cette orientation conservatrice, voire passéiste, met en relation les textes de diverses époques dans un constant brassage des interprétations qui participe, lui aussi, à l'intrication des différents plans dans lesquels s'inscrivent ces multiples histoires du droit. La perspective positiviste ne conduit pas à un appauvrissement du champ de vision au profit de la seule histoire normative, mais à un jeu de miroirs entre ces différents terrains avec un effet de kaléidoscope que vient renforcer la pluralité des points de vue des observateurs.

\section{III.2. Renouvellement perpétuel des modalités de l'enquête historique}

La conjonction d'une conception positiviste du droit et de l'histoire, que l'on trouve notamment chez Max Weber, ne peut être séparée du relativisme axiologique qui accorde une place de premier plan au point de vue subjectif de l'historien et au choix qu'il fait de ses moyens d'approche des phénomènes du passé. Chacun est convaincu aujourd'hui que le sujet écrivant l'histoire le fait à partir des questionnements propres à son contexte et à lui-même. Cette part inévitable, et enri-

49. Jean-Pierre BAuD, L'affaire de la main volée. Une histoire juridique du corps, Paris : Le Seuil, 1993. 
chissante, de subjectivité n'est pas contradictoire avec la recherche rigoureuse de moyens objectifs - c'est-à-dire soumis à l'évaluation et à la critique d'autrui - pour comprendre des phénomènes advenus dans le passé. C'est un truisme d'affirmer qu'il y autant d'histoires du droit que d'historiens du droit et même d'enquêtes liées à un terrain déterminé. Autant nous ne croyons guère au "pluralisme » juridique, exaltant l'irréductible diversité des expériences juridiques liées à chaque culture, autant nous plaidons pour une pluralité d'histoires du droit.

L'historien du droit travaille, pour l'essentiel, avec des textes d'un passé lointain ou proche 50 . À la différence de l'archéologue, il est relativement peu informé par les fouilles de monuments. Par ailleurs, l'éventuel recours à l'enquête orale, largement utilisée par les sociologues, n'est par définition possible que pour l'histoire du temps présent. Nous serions d'ailleurs tenté de voir dans cette question d'accès aux sources la ligne de partage la plus convaincante entre histoire et sociologie du droit. L'historien du droit ne cherche pas à interpréter ces textes relevant du champ juridique au sens large comme le fait le juriste praticien ou savant pour résoudre des problèmes concrets d'aujourd'hui, mais pour comprendre ce qui était en jeu dans le terrain d'enquête qu'il a choisi dans le passé.

Si l'on tient compte du fait qu'un grand nombre des textes utilisés par les historiens du droit ont été commentés de très nombreuses fois - ce qui n'empêche pas la découverte d'inédits ou l'exploitation de fonds archivistiques jusque-là négligés du fait de leur grande ampleur (notamment dans les domaines de l'histoire judiciaire et notariale) -, la recherche ne peut progresser que par la nouveauté des questionnements. Pour éviter l'écueil de l'orthodoxie historique, nous pensons de nouveau nécessaire la séparation entre la recherche historique et la dogmatique - même si rien n'interdit à la même personne de se livrer, en "changeant de casquette » aussi clairement que possible, aux deux activités. Se pose ensuite la question très controversée du regard porté par l'enquêteur que certains souhaitent "externe modéré ", tandis que d'autres le voudraient radicalement extérieur. Autrement dit, l'historien du droit doit-il être juriste, pour mieux comprendre la technique et le discours juridiques, ou vaut-il mieux qu'il évite de l'être, pour ne pas être prisonnier (y compris à son corps défendant) des préjugés des juristes ? Ce débat classique, opposant traditionnellement la corporation des historiens du droit et celles des sociologues ou des historiens généralistes, nous parait assez vain. N'attend-on pas de l'historien qu'il soit bien informé sur les spécificités de son terrain d'enquête, que l'historien des techniques navales ne soit pas étranger au monde de la mer ou que l'historien du commerce ne soit pas réfractaire à l'analyse économique ? À chacun de se doter des compétences qu'il estime nécessaires à la meilleure compréhension des textes qu'il étudie et d'essayer de se rendre indépendant des préjugés que sa formation est susceptible d'avoir fait naître dans son appréhension du droit. Il y a, selon nous, autant de difficultés pour le juriste et le non-juriste à pénétrer les techniques juridi-

50. Alain SUPIOT, « Rapport de synthèse de l'atelier 6 : interaction de la recherche juridique et de la recherche opérée dans les autres sciences sociales ", in Brigitte STERN (dir.), Livre blanc des Assises nationales de la recherche juridique, Paris : LGDJ, 1994, p. 65-66, parle d'un " patrimoine de textes ". 
ques du passé et autant de précautions à prendre pour s'affranchir des illusions des initiés ou des préventions des profanes.

L'historien du droit spécialisé devrait être amené à tenir compte le plus possible de ses compétences techniques, susceptibles de s'appliquer à des périodes historiques diverses, mais incapables de lui donner le spectre d'un historien "généraliste " attaché à l'étude d'une des grandes ères historiques et/ou linguistiques ${ }^{51}$. S'il est amené à se servir du vocabulaire propre au droit, il doit affronter les mêmes questions que tout historien sur l'application de la langue contemporaine et des instruments des sciences sociales à l'étude de cultures anciennes. Rien ne lui interdit de recourir aux concepts juridiques de son époque s'il les juge aptes à expliquer des phénomènes d'un passé plus ou moins lointain ignorant un tel vocabulaire notionnel ${ }^{52}$. La " barrière des mentalités » ne doit pas nous empêcher de « faire parler » les textes rédigés il y a plusieurs siècles ${ }^{53}$. Les pratiques autoréférentielles propres aux juristes - la citation des autorités, les réinterprétations successives des mêmes textes - ne sont pas en elles-mêmes des obstacles à l'herméneutique, mais au contraire les sources de continuelles remises en perspective.

Avec le point de vue propre à chaque historien, l'approche de tel ou tel champ juridique du passé varie, enfin, selon le terrain délimité (nous retrouvons ici la pluralité des objets), en fonction des sources utilisées (et de leur abondance relative par rapport à celles qui ne relèvent pas du droit; ainsi les textes de droit romain sont d'une ampleur limitée, mais ils nous font connaître la société romaine autant sinon mieux que d'autres témoignages) et de l'angle d'ouverture choisi en faisant appel ou non à d'autres sciences historiques. Là encore, les historiens du droit sont aujourd'hui plus sensibles que leurs prédécesseurs aux différences entre microhistoire et macro-histoire, entre analyse serrée ou en profondeur ( thick description", selon l'expression de Clifford Geertz ${ }^{54}$ ) et histoire comparative faisant appel à la technique de l'idéal-type ${ }^{55}$, ou encore entre «Isolierung» des représentations juridiques 56 et histoire du droit «intégrale» prétendant rendre compte des pratiques sociales autour du droit ${ }^{57}$. L'histoire du droit se renouvelle par la diversité même de ces angles d'attaque, par la comparaison entre plusieurs espaces ou plusieurs périodes, enfin par la possibilité même de se déplacer d'un point de vue ou d'un débat à l'autre 58. Quand Maitland parlait d’un tissu sans coutures ( a seam-

51. Yan THOMAS, « Histoire et Droit. Présentation », Annales ESC, 57(6), 2002, p. 1425-1428.

52. Michel Troper, "Sur l'usage des concepts juridiques en histoire", Annales. Économies, Sociétés, Civilisations, 47, 1992, p. 1171-1183.

53. Geoffrey Ernest Richard LLoY,, Pour en finir avec les mentalités, Paris : La Découverte, 1996

54. Clifford GEERTZ, "Thick Description», in ID., The Interpretation of Cultures, New York: Basic Books, 1973, p. 3-30.

55. Stephen KALBERG, La sociologie historique comparative de Max Weber (trad. fr. Hervé Maury), Paris : La Découverte, 2002.

56. Yan Thомаs, Mommsen et «L'Isolienung" du droit (Rome, l'Allemagne et l'État), Paris: de Boccard, 1984.

57. Reinhart KoseLLECK, L'expérience de l'histoire (trad. fr. Alexandre Escudier), Paris : Gallimard, Le Seuil, 1997, p. 180.

58. Andrew ABвOTT, Methods of Discovery: Heuristics for the Social Sciences, New York, Londres: W. W. Norton \& Co, 2004, p. 183 parle à ce sujet de " fractal heuristic». 
less $w e b »)$ à propos de l'histoire du droit anglais, il ne s'agissait pas, comme on le croit souvent, d'exalter la continuité historique du common law, mais de souligner l'arbitraire d'une méthode historique obligée de découper parmi les phénomènes du passé 59. Cette servitude de l'historien du droit, comme de tout chercheur en sciences sociales, est aussi une chance pour labourer de nouveau les mêmes champs d'études. Plus que jamais le pluriel s'impose pour des histoires du droit qui tentent de comprendre des phénomènes juridiques changeants, en allant au-delà de la fausse impression d'immobilisme ou de continuité des mondes du droit si souvent cultivée par les juristes eux-mêmes. C'est en s'aventurant dans les zones frontières à l'intérieur du champ juridique et en se risquant dans les périodes de rupture que le renouvellement de l'histoire du droit peut avoir le plus d'effets sur notre compréhension des phénomènes juridiques les plus contemporains comme les plus anciens.

\section{D'auteur}

Jean-Louis Halpérin est professeur à l'École normale supérieure (Paris) et membre de l'UMR 7074 «Centre de théorie et analyse du droit». Historien du droit, ses recherches portent sur la codification, la circulation des droits et les cultures des juristes. Parmi ses publications:

- Profils des mondialisations du droit, Paris : Dalloz, 2009;

- Histoire du droit des biens, Paris : Economica, 2008 ;

- Histoire des droits en Europe de 1750 à nos jours, Paris : Flammarion, 2004.

59. Frederic William MAITLAND, "A Prologue to a History of English Law », Law Quarterly Review, 14, 1898, p. 13. 
